\title{
Determinación de plaguicidas organoclorados en hortalizas del sur de Sonora: calidad y seguridad de los alimentos en relación a los límites máximos permitidos
}

\author{
Determination of organochlorine pesticides in vegetables grown in the south of Sonora: quality and \\ safety of foods in relation to the maximum permissible limits
}

\begin{abstract}
Paola Carolina Cantú Nava², María Mercedes Meza Montenegro², Ana Isabel Valenzuela Quintanar ${ }^{1}$ Claudia Osorio Rosas $^{2}$, Helga García Zamorano ${ }^{2}$, Patricia Grajeda Cota ${ }^{1}$, y María de Lourdes Gutiérrez Coronado ${ }^{1 *}$

Centro de Investigación en Alimentación y Desarrollo, A.C. Ciencia de los Alimentos. Carretera a la Victoria Km. O.6. Hermosillo, Sonora, 83000, México. Tel: 6622892400.

2 Instituto Tecnológico de Sonora. Departamento de Fuentes Naturales. 5 de febrero 818 Sur, Zona Centro, 85000 Cd. Obregón, Sonora, México.
\end{abstract}

\section{RESUMEN}

En la zona sur del estado de Sonora, México se localizan los Valles del Yaqui y Mayo, una de las áreas agrícolas más productivas del estado, donde el empleo de plaguicidas ha sido práctica común desde los inicios de la Revolución Verde en el año 1960. Su utilización puede ocasionar la presencia de residuos en hortalizas, con riesgo tanto para la salud humana como para su comercialización. El objetivo del presente estudio fue evaluar la presencia de residuos de plaguicidas organoclorados (POC), por su alta persistencia y bioacumulación $(\mathrm{HCH}$, hexaclorobenceno, lindano, heptacloro, aldrín, isodrín, heptacloro epóxido, gama y alfa clordano, endosulfán, dieldrín, DDE, endrín, DDD, DDT, metoxicloro, mirex), en papa, calabaza y chile. Se localizaron seis puntos de muestreo en cada uno de los valles y se estandarizó la metodología de cromatografía de gases. Los resultados indican que se encontraron cinco de los diecisiete plaguicidas analizados: endosulfán, DDT, dieldrín, lindano y $\mathrm{HCH}$ en las hortalizas muestreadas. El plaguicida predominante fue el endosulfán $(0.66-17.18 \mu \mathrm{g} / \mathrm{kg})$. Los resultados muestran la presencia de POC que se encuentran actualmente restringidos. No obstante, las concentraciones obtenidas no sobrepasaron los límites máximos permitidos para hortalizas establecidos por el Codex alimentarius.

Palabras claves: contaminantes orgánicos persistentes, cromatografía de gases, hortalizas.

\section{ABSTRACT}

The Mayo and Yaqui Valleys are located in the southern part of the state of Sonora, Mexico. It constitutes one of the most productive agricultural areas of the state, where the use of a wide range of pesticides has been a common practice since the beginning of the Green Revolution in 1960. Their use can lead to the presence of residues in vegetables, at risk for both human health and commercialization. The objective of the present study was to determine organochlorine pesticide residues (POC), due to its high persistence and bioaccumulation (Alpha-HCH, hexachlorobenzene, lindane, heptachlor, aldrin, isodrin, heptachlor epoxide, chlordane gamma, endosulfan, dieldrin, DDE, endrin, DDD, Alpha chlordane, DDT, methoxychlor, mirex) in potato, squash and chile, to determine their quality. Six sampling points in each of the valleys were located and a chromatographic methodology was validated. The results indicate that five of the seventeen pesticides analyzed were found: endosulfan, pp'-DDT, dieldrin, lindane and alpha-HCH in the vegetables sampled. The most predominant pesticide was endosulfan $(0.66-17.18 \mu \mathrm{g} / \mathrm{kg})$, because it is still used in the cultivation of vegetables. The results show the presence of POC that are currently restricted. However, the concentrations obtained from the pesticides did not exceed the concentrations of the maximum permissible limits for vegetables established by Codex alimentarius.

Key words: persistent organic pollutants, gas chromatography, vegetables.

\section{INTRODUCCIÓN}

La zona sur del estado de Sonora, comprendida por los Valles del Yaqui y Mayo, es una región agrícola por excelencia donde el uso de plaguicidas se ha convertido en una necesidad básica en los cultivos de frutas y hortalizas, convirtiéndola en una región potencialmente contaminada por diversos plaguicidas entre ellos los plaguicidas organoclorados (POC), que han sido ampliamente utilizados en la agricultura de esta región desde el inicio de la Revolución Verde (Moreno, 2008; Cantú et al., 2011; Valenzuela et al., 2015; Garca- Hernández et al., 2017).

La utilización inapropiada de los plaguicidas, particularmente su uso en exceso, su aplicación en tiempos no adecuados y en cultivos en los que no han sido registrados, hacen de estas sustancias un riesgo potencial para la salud humana y para el ambiente en general, ya que se trata de productos generalmente tóxicos que no solo contaminan el agua, el suelo y el aire, sino que también se acumulan en cultivos como de frutas y hortalizas, y que pueden permanecer como residuos en el producto final que va al consumidor $y$ por tanto, reducen su calidad (Fenik et al., 2011).

En México, a pesar de que, en el sector agrícola, se han prohibido varios plaguicidas, todavía se autorizan y utilizan algunos que están prohibidos en otros países; tal es el caso

*Autor para correspondencia: María de Lourdes Gutiérrez Coronado Correo electrónico: lulu@ciad.mx

Volumen XXI, Número 2 
del paraquat, lindano, paratión, malatión y endosulfán (González et al., 2010; Leyva et al., 2014; Bejarano, 2017; Benítez et al., 2018).

Los plaguicidas organoclorados son un conjunto de sustancias que poseen gran toxicidad, son altamente persistentes en el ambiente, no son solubles en agua, requieren de varios años o décadas antes de degradarse a formas menos dañinas, se evaporan y viajan grandes distancias en el aire y en el agua. Se pueden encontrar en ecosistemas terrestres y acuáticos, acumulándose principalmente en tejido graso, por lo que tienden a encontrarse en los distintos eslabones de la cadena alimentaria. Estas propiedades, y principalmente su libre circulación a través del efecto saltamontes, hacen que la presencia de los POC represente una amenaza global (De Boer y Wester, 1991). La utilización del DDT por casi 50 años y la persistencia de esta sustancia en el ambiente se refleja en los niveles de $\mathrm{pp}^{\prime}$-DDE, su principal metabolito, que se encuentra actualmente en el suero, tejido adiposo y leche materna de individuos no expuestos ocupacionalmente a dicho insecticida (Meza et al., 2013; Limón et al., 2017). La principal fuente de exposición a estos compuestos puede ser la ingesta de alimentos contaminados (Koepke et al., 2004). Las características previamente descritas clasifican a los plaguicidas organoclorados como plaguicidas altamente peligrosos, de acuerdo a los criterios establecidos por la FAO y OMS en 2008 y por la organización PAN (Pesticide Action Network, por sus siglas en inglés) en 2015 (Bejarano, 2017). En la actualidad se ha permitido nuevamente el uso restringido del DDT para el combate al paludismo y otras enfermedades transmitidas por vectores, en gran parte de África, el sur de Asia y América Latina (Pirsaheb et al., 2015).

El empleo de los plaguicidas en las actividades agrícolas, se hace conforme a normas de inocuidad, las cuales establecen límites máximos permitidos de residuos (LMP) para que el riesgo a corto y mediano plazo en los consumidores sea mínimo. El Codex alimentarius contribuye, a través de sus normas, directrices y códigos de prácticas alimentarias internacionales, a la inocuidad, la calidad y la equidad en el comercio internacional de alimentos. Por lo que el monitoreo y cuantificación de los POC en los alimentos se hace necesaria para corroborar su adecuado uso y determinar la calidad y seguridad de los alimentos (Codex alimentarius, 2018). Existen pocos estudios reportados en hortalizas en el Noroeste de México, donde resalta el de Valenzuela et al. (2008), analizando residuos de plaguicidas organoclorados en diferentes variedades de chiles, uva, tomate, agua y suelo en los valles del Yaqui, Mayo y Guaymas. Las variedades de chile, con excepción de chile serrano y morrón naranja, presentaron residuos de plaguicidas organoclorados (DDE 0.41-2.02 $\mu \mathrm{g} /$ $\mathrm{kg}$ ); y en chile caribe y Anaheim también se encontró endrín $(0.76$ y $0.18 \mu \mathrm{g} / \mathrm{kg})$. El tomate presentó residuos de DDE $(1.82-2.58 \mu \mathrm{g} / \mathrm{kg})$ y en uva, agua y suelo no se encontraron residuos. Asimismo, se han reportado, residuos de DDT en granos de trigo, frijol, maíz y garbanzo menores a los $20 \mu \mathrm{g} /$ kg (Aldana et al., 2008), de BHC, DDE, endrín, DDT y DDD en concentraciones entre $197-292 \mu \mathrm{g} / \mathrm{kg}$ en suelo agrícola del sur de Sonora (Cantú et al., 2011), endosulfán, DDE y DDT en concentraciones de 3-30 $\mu \mathrm{g} / \mathrm{kg}$ en agua (Verdugo, 2012). Así mismo, Meza et al. (2013), reportaron la presencia de pp'-DDE en muestras de sangre de niños del Valle del Yaqui y Mayo (0.25-10.3 $3 \mu \mathrm{g} / \mathrm{kg})$; y en menor proporción lindano, pp'-DDT, aldrín y endosulfán. Leal-Soto et al. (2014) reportaron la presencia de 17 POC en suelos agrícolas del centro y norte de Sonora, con mayores concentraciones de DDE $(0.07 \mu \mathrm{g} /$ $\mathrm{kg}$ ) en el Valle de Guaymas. De acuerdo a García Hernández et al. (2018), en México solo el 6 \% de la información reportada en la literatura corresponde a residuos de plaguicidas en alimentos, lo que evidencia la necesidad de implementar monitoreos constantes en estas matrices o en otras que nos permitan evaluar el riesgo de estos contaminantes en la salud y el medio ambiente.

El objetivo del presente estudio fue evaluar la presencia de residuos de plaguicidas organoclorados $(\mathrm{HCH}$, hexaclorobenceno, lindano, heptacloro, aldrín, isodrín, heptacloro epóxido, gama y alfa clordano, endosulfán, dieldrín, $\mathrm{DDE}$, endrín, DDD, DDT, metoxicloro, mirex), en papa, calabaza y chile cultivados en el sur de Sonora, determinados por cromatografía de gases, para conocer su calidad en relación a los límites máximos permisibles establecidos en el Codex Alimentarius.

\section{MATERIALES Y MÉTODOS Selección de la zona de estudio}

De acuerdo a la información recabada visitando las oficinas del Distrito de Riego del Rio Yaqui (DDR 041) en Ciudad Obregón y las de SAGARPA en la localidad de Navojoa, los cultivos más importantes y con mayor superficie de siembra para los Valles del Yaqui y Mayo son: papa, chile jalapeño y calabaza. Tomando como criterio de inclusión una mayor superficie de siembra de estas hortalizas, se seleccionaron seis campos en cada valle para lograr un total de doce sitios de muestreo distribuidos en la parte sur del estado de Sonora. Las muestras de hortalizas estudiadas se obtuvieron previa autorización de los productores.

\section{Muestreo}

El muestreo se llevó a cabo en el mes de mayo del 2012. Los sitios de muestreo, seis campos por cada valle, se ubicaron con ayuda del equipo GPS (Google Earth, 2012). En cada campo agrícola se trazaron cuatro puntos en forma de "Z", los cuales se establecieron por coordenadas geográficas con el apoyo de mapas obtenidos con el programa Google Earth (2011) versión 6.0.3.2197, tomando en cuenta que los puntos muestreados quedaran distribuidos en todo el campo (Tabla 1).

\section{Toma de muestra}

Las muestras fueron recolectadas siguiendo la metodología descrita por la Norma Mexicana NMX-AA132-SCFI-2006. La hortaliza muestreada fue la cultivada en el campo agrícola establecido por el GPS, donde se puede encontrar cualquiera de las hortalizas seleccionadas para el 
Tabla 1. Hortalizas muestreadas en los campos agrícolas seleccionados en los Valles del Yaqui y Mayo en Sonora, México.

Table 1. Vegetables sampled in selected agricultural fields in the Yaqui and Mayo Valleys in Sonora, Mexico.

\begin{tabular}{|c|c|c|c|c|c|}
\hline Hortaliza & Punto & Muestra 1 & Muestra 2 & Muestra 3 & Muestra 4 \\
\hline Calabaza & P1 & $\begin{array}{c}\text { N } 27^{\circ} 28^{\prime} 25.6^{\prime \prime} \\
\text { W } 109^{\circ} 59^{\prime} 40.3^{\prime \prime}\end{array}$ & 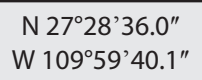 & $\begin{array}{c}\text { N } 27^{\circ} 28^{\prime} 36.0 \\
\text { W } 110^{\circ} 00^{\prime} 12.9^{\prime \prime}\end{array}$ & $\begin{array}{c}\text { N } 27^{\circ} 28^{\prime} 24.6^{\prime \prime} \\
\text { W } 1110^{\circ} 00^{\prime} 15.2^{\prime \prime}\end{array}$ \\
\hline Calabaza & P3 & $\begin{array}{l}\text { N } 27^{\circ} 28^{\prime} 24.2^{\prime \prime} \\
\text { W } 110^{\circ} 13^{\prime} 24.7^{\prime \prime}\end{array}$ & $\begin{array}{l}\text { N } 27^{\circ} 28^{\prime} 35.3^{\prime \prime} \\
\text { W } 110^{\circ} 13^{\prime} 24.4^{\prime \prime}\end{array}$ & 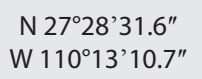 & $\begin{array}{l}\text { N } 27^{\circ} 28^{\prime} 31.6^{\prime \prime} \\
\text { W } 110^{\circ} 13^{\prime} 11.1^{\prime \prime}\end{array}$ \\
\hline Papa & P5 & $\begin{array}{l}\text { N } 27^{\circ} 24^{\prime} 02.0^{\prime \prime} \\
\text { W } 110^{\circ} 02^{\prime} 0.50^{\prime \prime}\end{array}$ & $\begin{array}{l}\text { N } 27^{\circ} 26^{\prime} 44.3^{\prime \prime} \\
\text { W } 110^{\circ} 011^{\prime} 52.4^{\prime \prime}\end{array}$ & $\begin{array}{c}\text { N } 27^{\circ} 24^{\prime} 33^{\prime \prime} \\
\text { W } 110^{\circ} 011^{\prime} 31.1^{\prime \prime}\end{array}$ & $\begin{array}{c}\text { N } 27^{\circ} 24^{\prime} 1.3^{\prime \prime} \\
\text { W } 110^{\circ} 01^{\prime} 35.1^{\prime \prime}\end{array}$ \\
\hline Papa & P6 & $\begin{array}{l}\text { N } 27^{\circ} 18^{\prime} 40.3^{\prime \prime} \\
\text { W } 109^{\circ} 55^{\prime} 55.3^{\prime \prime}\end{array}$ & 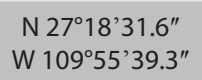 & $\begin{array}{c}\text { N } 27^{\circ} 18^{\prime} 55.3^{\prime \prime} \\
\text { W } 109^{\circ} 55^{\prime} 39.3^{\prime \prime}\end{array}$ & $\begin{array}{l}\text { N } 27^{\circ} 18^{\prime} 55.4^{\prime \prime} \\
\text { W } 109^{\circ} 55^{\prime} 53.8^{\prime \prime}\end{array}$ \\
\hline Chile & P7 & $\begin{array}{l}\text { N } 27^{\circ} 03^{\prime} 80.7^{\prime \prime} \\
\text { W } 109^{\circ} 46^{\prime} 76.6^{\prime \prime}\end{array}$ & 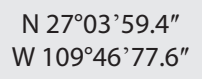 & $\begin{array}{l}\text { N 27003'58.7" } \\
\text { W } 109^{\circ} 47^{\prime} 19.9^{\prime \prime}\end{array}$ & $\begin{array}{l}\text { N } 27^{\circ} 03^{\prime} 76.1^{\prime \prime} \\
\text { W } 109^{\circ} 46^{\prime} 95.7^{\prime \prime}\end{array}$ \\
\hline Chile & P9 & $\begin{array}{l}\text { N } 26^{\circ} 99^{\prime} 81.5^{\prime \prime} \\
\text { W } 109^{\circ} 56^{\prime} 18.9^{\prime \prime}\end{array}$ & $\begin{array}{l}\text { N 26⒐99'82.6" } \\
\text { W 109'56'78.7" }\end{array}$ & $\begin{array}{l}\text { N } 26^{\circ} 99^{\prime} 40.8^{\prime \prime} \\
\text { W } 109^{\circ} 56^{\prime} 80.3^{\prime \prime}\end{array}$ & $\begin{array}{l}\text { N } 26^{\circ} 99^{\prime} 42.3^{\prime \prime} \\
\text { W } 109^{\circ} 56^{\prime} 15.1^{\prime \prime}\end{array}$ \\
\hline Papa & P10 & $\begin{array}{l}\text { N 26 } 26^{\circ} 53^{\prime} 37.4^{\prime \prime} \\
\text { W } 109^{\circ} 36^{\prime} 31.2^{\prime \prime}\end{array}$ & $\begin{array}{l}\text { N } 26^{\circ} 53^{\prime} 37.3^{\prime \prime} \\
\text { W } 109^{\circ} 36^{\prime} 45^{\prime \prime}\end{array}$ & $\begin{array}{l}\text { N 26 } 26^{\circ} 3^{\prime} 49.4^{\prime \prime} \\
\text { W } 109^{\circ} 36^{\prime} 29.8^{\prime \prime}\end{array}$ & $\begin{array}{l}\text { N } 26^{\circ} 54^{\prime} 01.7^{\prime \prime} \\
\text { W } 109^{\circ} 36^{\prime} 41.7^{\prime \prime}\end{array}$ \\
\hline Calabaza & P11 & $\begin{array}{l}\text { N } 26^{\circ} 50^{\prime} 24.7^{\prime \prime} \\
\text { W } 109^{\circ} 37^{\prime} 49.9^{\prime \prime}\end{array}$ & $\begin{array}{l}\text { N } 26^{\circ} 50^{\prime} 11.10^{\prime \prime} \\
\text { W } 109^{\circ} 37^{\prime} 36^{\prime \prime}\end{array}$ & $\begin{array}{l}\text { N 2650'11.1" } \\
\text { W 109³7'22.4" }\end{array}$ & $\begin{array}{l}\text { N } 26^{\circ} 50^{\prime} 20.0^{\prime \prime} \\
\text { W } 109^{\circ} 37^{\prime} 22.5^{\prime \prime}\end{array}$ \\
\hline Calabaza & P12 & $\begin{array}{l}\text { N } 26^{\circ} 50^{\prime} 14.9^{\prime \prime} \\
\text { W } 109^{\circ} 30^{\prime} 52.9^{\prime \prime}\end{array}$ & $\begin{array}{l}\text { N 2650'17.7" } \\
\text { W 109 } 30^{\prime} 52.9^{\prime \prime}\end{array}$ & $\begin{array}{l}\text { N 2650'17.7" } \\
\text { W 109³0'21.8" }\end{array}$ & $\begin{array}{l}\text { N 26 } 26^{\circ} 50^{\prime} 14.6^{\prime \prime} \\
\text { W } 109^{\circ} 30^{\prime} 22.0^{\prime \prime}\end{array}$ \\
\hline
\end{tabular}

presente estudio, como se muestra en la Tabla 1. Los Puntos de muestreo del 1 al 6 corresponden al Valle del Yaqui y los del 7 al 12 al Valle del Mayo.

Se tomaron cuatro muestras por campo agrícola, obteniéndose un total de 48 muestras para analizar. Se muestreó $1 / 2 \mathrm{~kg}$ de hortaliza utilizando guantes para evitar alguna interferencia. Se emplearon bolsas de papel, previamente etiquetadas con los siguientes datos: sitio de muestreo, punto de muestreo, hora, fecha y nombre del muestreador. Las muestras se trasladaron al laboratorio a temperatura ambiente, donde fueron procesadas, sub-muestreadas en porciones de $250 \mathrm{~g}$ y almacenadas en un congelador a una temperatura de $-40^{\circ} \mathrm{C}$ hasta su análisis.

\section{Análisis cromatográfico de los plaguicidas organoclora- dos}

El análisis cromatográfico de los plaguicidas a- $\mathrm{HCH}$, lindano, heptacloro, aldrín, isodrín, heptacloro epóxido, a-clordano, $y$-clordano, endosulfán, dieldrín, DDE, endrín, DDD, DDT, metoxicloro, mirex y hexaclorobenceno; se llevó a cabo en un cromatógrafo de gases.

\section{Características del equipo}

Se utilizó un cromatógrafo de gases (CG) Agilent Technologies 7890A (Network GC system) equipado con un automuestreador 7683B y un detector de microcaptura de electrones $(\mu E C D)$ con una fuente radioactiva de Ni-63, y con una muy alta sensibilidad. Se utilizó una columna de polaridad media DB-5 con $30 \mathrm{~m}$ de longitud, $0.250 \mathrm{~mm}$ de diámetro interno y $0.25 \mu \mathrm{m}$ de la película. El gas acarreador en la columna fue helio. Las condiciones de trabajo fueron las siguientes: T del inyector $270^{\circ} \mathrm{C}$, T de la columna $280^{\circ} \mathrm{C}$, T del detector $340^{\circ} \mathrm{C}$. Control de la temperatura: T inicial de $110^{\circ} \mathrm{C}$ (1 min) T final $280^{\circ} \mathrm{C}$ ( 2 min con incrementos de $15^{\circ} \mathrm{C} / \mathrm{min}$ ).

\section{Aseguramiento de la calidad de los datos}

Para cada corrida de extracción de plaguicidas organoclorados en la matriz de hortaliza, se corrió un triplicado de muestra fortificada, una muestra de hortaliza libre de plaguicidas, un blanco reactivo y un blanco cristalería, además un duplicado de extracción de una muestra problema.

\section{Estandarización del Método Dispersión de Matriz en Fase Sólida (DMFS)}

Con la finalidad de analizar los residuos de POC en las hortalizas muestreadas, se estandarizó en el laboratorio la técnica de dispersión en matriz en fase sólida (DMFS), validada por Valenzuela et al. (2006), con modificaciones para la extracción de POC en hortalizas. Para asegurar que se obtuvieran datos confiables, se determinaron los siguientes parámetros: Linealidad del sistema y linealidad del método, exactitud (\% $\mathrm{R})$, precisión (CV). También se obtuvieron los límites de detección del equipo (LD) y los límites de cuantificación (LC). 


\section{Linealidad del sistema}

Para medir la linealidad del sistema cromatográfico se realizó una curva de calibración para cada plaguicida compuesta por siete niveles de concentración $(50,75,100$, $125,150,175$ y $200 \%$ ) considerando el $100 \%$ como el límite máximo permitido de residuos de plaguicidas. La curva compuesta por las concentraciones mencionadas anteriormente se preparó tres días consecutivos y se inyectó cada curva por triplicado para obtener el coeficiente de regresión de cada plaguicida.

\section{Linealidad del método}

Para evaluar la linealidad del método se fortificaron con $100 \mu \mathrm{L}$ muestras blanco a diferentes niveles de concentración (50, 75, 100, 125, 150, 175 y $200 \%$ ) de la mezcla de plaguicidas organoclorados. Posteriormente se realizó la extracción de los fortificados por tres días consecutivos inyectándose al C G por triplicado. Por último, de los cromatogramas obtenidos se graficó el área contra la concentración de cada plaguicida obteniéndose el coeficiente de regresión (R2).

\section{Exactitud}

El parámetro de exactitud se determinó comparando las concentraciones de los plaguicidas de las muestras de hortaliza fortificadas y las concentraciones de las soluciones de trabajo ( $50 \%, 75 \%, 100 \%, 125 \%, 150 \%, 175 \%$ y $200 \%)$. Se realizó una serie de fortificados por cada lote de muestras analizadas, de los cuáles se obtuvo el porcentaje de recuperación con los datos obtenidos al ser leídos por el detector del cromatógrafo de gases.

\section{Precisión}

Para determinar el parámetro de precisión se calculó la desviación estándar de los porcentajes de recuperación de las muestras adicionadas y el coeficiente de variación promedio entre los fortificados de las muestras de hortaliza.

\section{Límite de Detección}

Para obtener el límite de detección se realizaron inyecciones al C G de concentraciones menores de los estándares de POC con el fin de determinar la concentración mínima sensible al detector.

\section{Límite de Cuantificación}

El límite de cuantificación se determinó realizando inyecciones al C G de la misma manera que el límite de detección, con el fin de determinar la concentración mínima que se puede extraer.

\section{RESULTADOS Y DISCUSIÓN}

El método de dispersión de matriz en fase sólida fue estandarizado cumpliendo con los requisitos de calidad (exactitud, precisión y linealidad) establecidos por el Departamento de Agricultura de los E.E.U.U. (USDA) y por la Agencia de Protección Ambiental de los E.E.U.U. (EPA). Se obtuvieron los siguientes valores: Exactitud: 94.99-106.65, Precisión: 0.08-8.17 y Linealidad: 0.9784-0.9971. Así mismo se obtuvo un límite de detección de $2-20 \mu \mathrm{g} / \mathrm{kg}$ y un límite de cuantificación de 6.6-66.6 $\mu \mathrm{g} / \mathrm{kg}$.

\section{Análisis de plaguicidas organoclorados}

En la Tabla 2 se muestran los POC detectados en las muestras de hortalizas analizadas. De las 48 muestras analizadas sólo se encontró la presencia de al menos uno de los plaguicidas estudiados en 23 muestras, lo que representa un $47.9 \%$ del total de las muestras. De las muestras encontradas positivas a POC, 19 presentaron endosulfán (39.6\%), 5 dieldrín (10.4\%), 4 pp'-DDT (8.3\%), 2 alfa-HCH (4.1\%) y 2 lindano (4.1\%), lo que significa, que de los 17 plaguicidas analizados sólo fueron detectados cinco.

Tabla 2. Plaguicidas organoclorados detectados en muestras de hortalizas procedentes de los Valles del Yaqui y Mayo en Sonora, México.

Table 2. Organochlorine pesticides detected in vegetable samples from the Yaqui and Mayo Valleys in Sonora, Mexico.

\begin{tabular}{|lc|}
\hline Plaguicidas Organoclorados & \% de muestras positivas \\
\hline Alfa HCH & 4.1 \\
\hline Hexaclorobenceno & ND \\
\hline Lindano & 4.1 \\
\hline Heptacloro & ND \\
\hline Aldrín & ND \\
\hline Isodrín & ND \\
\hline Heptacloro epóxido & ND \\
\hline Gama Clordano & ND \\
\hline Endosulfán & 39.6 \\
\hline Dieldrín & 10.4 \\
\hline pp'-DDE & ND \\
\hline Endrín & ND \\
\hline Pp'-DDD & ND \\
\hline Alfa clordano & ND \\
\hline pp'-DDT & 8.3 \\
\hline Metoxicloro & ND \\
\hline Mirex & ND \\
\hline
\end{tabular}

$\mathrm{ND}=$ no detectado

El endosulfán se encontró en un $39.6 \%$ de las muestras, evidenciando que la hortaliza durante los días en que se realizó el muestreo, estuvo sometida a tratamientos con este plaguicida, ya que al ser rociado en la planta; el $50 \%$ del residuo desaparece en un periodo de tres a cinco días y se requiere un estimado de 90 días para su completa desaparición (Romero et al., 2018), o bien pudo haberse incorporado a la hortaliza a través del agua o el suelo (Leal-Soto et al., 2014, Valenzuela et al., 2015). Su presencia en los tres tipos de hortaliza analizados evidencia su gran utilización para el combate a plagas o enfermedades en el cultivo de distintos tipos de hortalizas. A partir del año 2016 la Comisión federal para la protección contra riesgos sanitarios, COFEPRIS, can- 
celó el registro de este plaguicida (Bejarano, 2017). Un $8.3 \%$ y $6.3 \%$ de las muestras presentaron pp'-DDT y dieldrin respectivamente; actualmente su uso se encuentra restringido, pero debido a su persistencia, todavía pueden detectarse en diferentes medios del ambiente, entre ellos el suelo agrícola (Ortiz, 2001; Leal-Soto et al., 2014; Valenzuela et al., 2015). La Agencia para las Sustancias Toxicas y el Registro de Enfermedades (ASTDR) evalúan la persistencia de las sustancias en el ambiente de acuerdo al parámetro Koc (coeficiente de adsorción). En esta calificación las sustancias con Koc superior a 100 mil son las de mayor adsorción, más de 10 mil adsorción media. El pp'-DDT cuenta con un Koc de 240 mil, por lo que presenta una alta afinidad al suelo, mientras que el dieldrín presenta un Koc de 12 mil (media). Dicha afinidad podría explicar su presencia en muestras de hortalizas, ya que este plaguicida se bioacumula en plantas por medio de las raíces, lo cual depende de la concentración del contaminante en el suelo (Valenzuela et al., 2015).

En un $6.3 \%$ de las muestras se presentó el alfa-HCH y lindano (gama-HCH), sus usos tuvieron auge para el combate de vectores tales como los piojos y termitas; por lo que podrían aún persistir en el suelo.

Las concentraciones de plaguicidas organoclorados encontrados en las muestras positivas de hortalizas procedentes de los Valles del Yaqui y Mayo se muestran en las
Tablas 3 y 4, así mismo, se muestran los límites máximos de residuos permisibles establecidos en el Codex alimentarius para hortalizas.

El endosulfán, presentó concentraciones en un rango de no detectado ND - $17.18 \mu \mathrm{g} / \mathrm{kg}$, y se encontró en los tres tipos de hortalizas analizadas, debido probablemente al gran uso que se le ha dado en diferentes tipos de cultivos agrícolas, y a la presencia de estos contaminantes en suelos agrícolas (Cantú et al., 2011; Leal-Soto et al., 2014; Valenzuela et al., 2015). El endosulfán en el suelo es moderadamente persistente y se acumula en muchos organismos expuestos a este plaguicida, sin embargo, no persiste en sus tejidos una vez que desaparece la fuente de exposición, ya que es metabolizado rápidamente en hígado y riñón para ser eliminado del cuerpo (Romero et al., 2018).

En las muestras de papa provenientes del Valle del Yaqui, se presentaron concentraciones del pp'-DDT en un rango de $3.22-5.14 \mu \mathrm{g} / \mathrm{kg}$. Estudios realizados por Giaunnizzi (1994), demuestran que la cáscara de la papa presenta características lipídicas que le brindan mayor afinidad por los plaguicidas. Debido a las características lipofílicas de los plaguicidas organoclorados, este tipo de hortaliza presenta gran disposición a la contaminación por parte de este tipo de compuestos. Lo que coincide con lo reportado por Nolasco y Zúniga (1992), quienes encontraron residuos de plaguicidas

Tabla 3. Concentraciones de Plaguicidas Organoclorados en muestras de hortalizas procedentes del Valle del Yaqui ( $\mu \mathrm{g} / \mathrm{kg})$.

Table 3. Concentrations of Organochlorine Pesticides in samples of vegetables from the Yaqui Valley $(\mu \mathrm{g} / \mathrm{kg})$.

\begin{tabular}{|c|c|c|c|c|c|c|c|c|c|c|c|c|c|}
\hline & P3M1 & P3M2 & P4M2 & P4M3 & P5M1 & P5M2 & P5M3 & P5M4 & P6M1 & P6M2 & P6M3 & P6M4 & \\
\hline & C & C & $\mathbf{C H}$ & CH & $\mathbf{P}$ & $\mathbf{P}$ & $\mathbf{P}$ & $\mathbf{P}$ & $\mathbf{P}$ & $\mathbf{P}$ & $\mathbf{P}$ & $\mathbf{P}$ & \\
\hline Alfa-HCH & 2.11 & 1.2 & ND & ND & ND & ND & ND & ND & ND & ND & ND & ND & 10 \\
\hline Hexaclorobenceno & ND & ND & ND & ND & ND & ND & ND & ND & ND & ND & ND & ND & - \\
\hline Lindano & ND & 1.08 & ND & ND & ND & ND & ND & ND & ND & ND & ND & ND & 10 \\
\hline Aldrín & ND & ND & ND & ND & ND & ND & ND & ND & ND & ND & ND & ND & 50 \\
\hline Isodrín & ND & ND & ND & ND & ND & ND & ND & ND & ND & ND & ND & ND & 50 \\
\hline Heptacloro epóxido & ND & ND & ND & ND & ND & ND & ND & ND & ND & ND & ND & ND & 20 \\
\hline Gama Clordano & ND & ND & ND & ND & ND & ND & ND & ND & ND & ND & ND & ND & 200 \\
\hline Endrín & ND & ND & ND & ND & ND & ND & ND & ND & ND & ND & ND & ND & 50 \\
\hline$P p^{\prime}-D D D$ & ND & ND & ND & ND & ND & ND & ND & ND & ND & ND & ND & ND & 200 \\
\hline Alfa clordano & ND & ND & ND & ND & ND & ND & ND & ND & ND & ND & ND & ND & 200 \\
\hline $\mathrm{pp}$-DDT & ND & ND & ND & ND & 5.14 & 4.4 & 3.5 & 3.22 & ND & ND & ND & ND & 200 \\
\hline Metoxicloro & ND & ND & ND & ND & ND & ND & ND & ND & ND & ND & ND & ND & 50 \\
\hline Mirex & ND & ND & ND & ND & ND & ND & ND & ND & ND & ND & ND & ND & 500 \\
\hline
\end{tabular}

$\mathrm{P}=$ punto de muestreo, $\mathrm{M}=$ número de muestra, $\mathrm{C}=$ calabaza, $\mathrm{CH}=$ chile, $\mathrm{P}=$ papa, $\mathrm{ND}=$ no detectado, LMP= Límite Máximo Permitido (Codex Alimentarius, 2011) 
Tabla 4. Concentraciones de Plaguicidas Organoclorados en muestras de hortalizas procedentes del Valle del Mayo $(\mu \mathrm{g} / \mathrm{kg})$.

Table 4. Concentrations of Organochlorine Pesticides in samples of vegetables from the Mayo Valley $(\mu \mathrm{g} / \mathrm{kg})$.

\begin{tabular}{|c|c|c|c|c|c|c|c|c|c|c|c|c|}
\hline & $\begin{array}{l}\text { P7M2 } \\
\text { CH }\end{array}$ & $\begin{array}{l}\text { P8M1 } \\
\text { P }\end{array}$ & $\begin{array}{c}\text { P8M2 } \\
\text { P }\end{array}$ & $\begin{array}{c}\text { P8M3 } \\
\text { P }\end{array}$ & $\begin{array}{l}\text { P8M4 } \\
\text { P }\end{array}$ & $\begin{array}{l}\text { P10M1 } \\
\text { P }\end{array}$ & $\begin{array}{l}\text { P10M2 } \\
\text { P }\end{array}$ & $\begin{array}{l}\text { P12M1 } \\
\text { P }\end{array}$ & $\begin{array}{l}\text { P12M2 } \\
\text { P }\end{array}$ & $\begin{array}{c}\text { P12M3 } \\
\text { C }\end{array}$ & $\begin{array}{l}\text { P12M4 } \\
\text { C }\end{array}$ & LMP \\
\hline Alfa-HCH & ND & ND & ND & ND & ND & ND & ND & ND & ND & ND & ND & 10 \\
\hline Hexaclorobenceno & ND & ND & ND & ND & ND & ND & ND & ND & ND & ND & ND & \\
\hline Lindano & ND & 1.08 & ND & ND & ND & ND & ND & ND & ND & ND & ND & 10 \\
\hline Heptacloro & ND & ND & ND & ND & ND & ND & ND & ND & ND & ND & ND & 20 \\
\hline Aldrín & ND & ND & ND & ND & ND & ND & ND & ND & ND & ND & ND & 50 \\
\hline Isodrín & ND & ND & ND & ND & ND & ND & ND & ND & ND & ND & ND & 50 \\
\hline Heptacloro epóxido & ND & ND & ND & ND & ND & ND & ND & ND & ND & ND & ND & 20 \\
\hline Gama Clordano & ND & ND & ND & ND & ND & ND & ND & ND & ND & ND & ND & 200 \\
\hline Endosulfán & 0.85 & ND & ND & 1.14 & 0.85 & 0.66 & 1.65 & 0.66 & 5.65 & 1.05 & 1.33 & 500 \\
\hline Dieldrín & ND & 0.84 & 1.29 & ND & 1.24 & ND & ND & ND & ND & ND & ND & 50 \\
\hline$p p^{\prime}-D D E$ & ND & ND & ND & ND & ND & ND & ND & ND & ND & ND & ND & 200 \\
\hline Endrín & ND & ND & ND & ND & ND & ND & ND & ND & ND & ND & ND & 50 \\
\hline$P p^{\prime}-D D D$ & ND & ND & ND & ND & ND & ND & ND & ND & ND & ND & ND & 200 \\
\hline Alfa clordano & ND & ND & ND & ND & ND & ND & ND & ND & ND & ND & ND & 200 \\
\hline $\mathrm{pp}^{\prime}-\mathrm{DDT}$ & ND & ND & ND & ND & ND & ND & ND & ND & ND & ND & ND & 200 \\
\hline Metoxicloro & ND & ND & ND & ND & ND & ND & ND & ND & ND & ND & ND & 50 \\
\hline Mirex & ND & ND & ND & ND & ND & ND & ND & ND & ND & ND & ND & 500 \\
\hline
\end{tabular}

$\mathrm{P}=$ punto de muestreo, $\mathrm{M}=$ número de muestra, $\mathrm{C}=$ calabaza, $\mathrm{CH}=$ chile, $\mathrm{P}=$ papa, $\mathrm{ND}=$ no detectado, $\mathrm{LMP}=$

Límite Máximo Permitido (Codex Alimentarius, 2011)

organoclorados en 39 muestras de hortalizas frescas analizadas, procedentes de una zona agrícola de Lepaterique, Feo Morazan en Honduras, siendo el pp'-DDT el principal compuesto detectado. Waliszewski et al. (2008), estudiaron plaguicidas organoclorados, como el lindano y DDT en zanahoria, encontrando que los POC se acumulan en mayor proporción en la piel que en la pulpa de esta hortaliza.

También, se obtuvieron concentraciones de dieldrín en muestras de papas pertenecientes al Valle del Mayo; dichas concentraciones se encuentran en un rango de no detectado ND- $1.29 \mu \mathrm{g} / \mathrm{kg}$; su presencia se debe a la degradación de aldrín en el medio ambiente, considerándose el dieldrín más resistente a la degradación (USEPA, 1979; Miglioranza et al., 2002). Así mismo, se detectó la presencia de lindano en una de las muestras, a una concentración de $1.08 \mu \mathrm{g} / \mathrm{kg}$. Sin embargo, la presencia de dieldrín y lindano no fue significativa, ya que se detectaron solo trazas.

El alfa-HCH se presentó solo en dos muestras de calabaza pertenecientes al Valle del Yaqui, en un rango de 1.20$2.11 \mu \mathrm{g} / \mathrm{kg}$, siendo su presencia también no significativa. CESCCO-IHISE (1997), en un estudio que cubrió 76 hortalizas procedentes de mercados y supermercados de Tegucigalpa y Comayagüela, en Honduras, el cual incluyó apio, cebolla blanca y roja, chile verde, coliflor, lechuga, pepino, papa, repollo, tomate y zanahoria, reportó la presencia de POC particularmente $\mathrm{pp}^{\prime}-\mathrm{DDT}$, endosulfán, heptacloro y lindano en un $82 \%$ de las muestras. Residuos de dieldrín, pp'-DDT y lindano han sido detectados en todos los monitoreos en cultivos de papa realizados en USA, Inglaterra, Japón y otros países (Giannuzzi, 1994).

Los resultados de este estudio muestran la presencia de POC en las hortalizas analizadas, una de las rutas por la cual estos contaminantes llegan a las hortalizas podría ser por su presencia en los suelos agrícolas. Estudios realizados en suelo agrícola y urbano del Valle del Yaqui, demostraron la presencia de endosulfán, endrín, aldrín, pp'-DDT y sus metabolitos (Osorio, 2008; Cantù et al., 2011; Rascon, 2011; Navarro, 2012). En cuanto al Valle del Mayo, Osorio (2008) y Acuña (2008), reportaron la presencia de hexaclorobenceno, lindano, endosulfán, $\mathrm{pp}^{\prime}-\mathrm{DDE}$, endrín, $\mathrm{pp}$ '-DDT y $\mathrm{pp}^{\prime}-\mathrm{DDD}$. También se ha reportado la presencia de POC en suelos del norte y centro de Sonora (Cantú et al., 2011; Leal-Soto et al., 2014; Valenzuela et al., 2015).

Las concentraciones obtenidas de los diferentes plaguicidas detectados en este estudio, se encontraron por debajo de los límites máximos de residuos permisibles establecidos por el Codex alimentarius para hortalizas, por lo que no representan algún riesgo para la salud del consumidor, ni para su comercialización.

Además, de las 48 muestras analizadas, 25 muestras (52\%) no presenta residuos de plaguicidas, lo que coincide con lo reportado por Pérez et al. (2013) y por Galt (2009) quien reportó que de 14,212 muestras de hortalizas que ingresaron a EUA, el $52 \%$ presentó residuos de algún plaguicida y que las concentraciones encontradas están por debajo de los LMP. Esto implica que, mediante la implementación de buenas prácticas agrícolas, un manejo integrado de plagas y enfermedades, y un buen uso y manejo de plaguicidas, 
se puede incrementar considerablemente el porcentaje de muestras sin residuos y, por ende, asegurar la calidad del producto y la seguridad para el mercado y el consumidor final. Sin embargo, es importante tomar en cuenta que son pocas las muestras y campos monitoreados, por lo que se recomienda escalar el estudio para obtener resultados más generalizados. Es importante el continuo monitoreo en hortalizas para constatar su calidad para el consumo por los mercados tanto nacional como el de exportación.

Los resultados del presente estudio contrastan con lo reportado por Ramirez-Milla (2009), quien mostró que los plaguicidas como el captán, se degrada a tetrahidroftalimida (THFI), carbofurán, endosulfán, malatión y metamidofos determinados en calabacita, tomate verde y tomate saladette rebasaron los LMP establecidos por la Comunidad Europea. En tomate verde, la concentración del metamidofos fue 15 veces el LMP, del endosulfán 10.9 veces y del malatión 1.3 veces. En tomate saladette, la concentración del metamidofos fue 42 veces el LMP, del THFI 258 veces y del malatión 1.9 veces. En calabaza el malatión fue 25.5 veces el LMP.

Aunque es de destacar que los LMP considerados en México por lo general son más altos que los establecidos por la Unión Europea o por el Codex Alimentarius; los cuales son similares a los de Canadá y EUA.

Estos contrastes evidencian que los productores de hortalizas en Sonora, al regirse principalmente mediante una agricultura contractual de exportación, asociada en gran medida a los mercados de EUA y la Unión Europea, repercute en las políticas de inocuidad y en una tendencia al uso de compuestos con menor persistencia y residualidad para evitar la devolución de sus productos, debido a que contengan residuos de plaguicidas (Moreno y López, 2005) que garanticen la calidad establecida en los mercados, y satisfagan a los consumidores que demandan un suministro constante de alimentos limpios, de alta calidad, sanos y seguros (Atreya, 2006).

De igual manera, se debe de considerar que a pesar de que en este estudio se encontraron concentraciones por debajo de los límites máximos permisibles, actualmente se tiene una exposición a numerosos productos químicos tanto en los alimentos, como en el agua, el aire y el suelo. Por lo que se sugiere como perspectiva científica la llamada "mezcla toxicológica", la cual se refiere a las complejas interacciones de los productos químicos y los efectos de exposiciones acumuladas, que al considerar un efecto aditivo el riesgo potencial se incrementa (van der Hoff y van Zoonen, 1999; Pérez et al., 2009; Borchers et al., 2010), por lo que se sugiere encaminar las futuras investigaciones en este sentido.

Este estudio se realizó durante el año 2012, por lo que los resultados encontrados se compararon con los LMP establecidos en el Codex Alimentarius del año 2011, donde se reportan los LMP para hortalizas en general. Para el año 2016, los valores reportan la asociación de plaguicida por cultivo de hortalizas. Para el caso de la papa se reportan valores para aldrín, dieldrín (100 $\mu \mathrm{g} / \mathrm{kg})$ y endosulfán $(50 \mu \mathrm{g} /$ kg). Comparando estos valores con los LMP del 2011, el valor se hace más estricto para el endosulfán que pasa de 500 a 50 $\mu \mathrm{g} / \mathrm{kg}$ y se hace más laxo para aldrín y dieldrín que pasa de 50 a $100 \mu \mathrm{g} / \mathrm{kg}$. Para la calabaza se tienen valores reportados para aldrín, dieldrín $(100 \mu \mathrm{g} / \mathrm{kg})$, endosulfán $(500 \mu \mathrm{g} / \mathrm{kg})$ y endrín $(50 \mu \mathrm{g} / \mathrm{kg})$. Los valores para endosulfán y endrín se mantienen iguales con respecto al 2011 y también se hacen más laxos para aldrín y dieldrín. Para el chile jalapeño solo se reportan valores para el endosulfán $(500 \mu \mathrm{g} / \mathrm{kg})$ el cual se mantiene igual con respecto al 2011. A pesar de los valores más estrictos en los LMP en el caso del endosulfán, las concentraciones encontradas en este estudio siguen estando por debajo de los LMP.

En México son contadas las propuestas de métodos para la cuantificación de residuos de plaguicidas en hortalizas; de igual forma, son pocos los estudios realizados en el monitoreo de residuos de plaguicidas en las hortalizas destinadas al mercado nacional. Generalmente, los laboratorios especializados para ello, se avocan al análisis de residuos contaminantes para las hortalizas destinadas a la exportación (Pérez et al., 2013).

\section{CONCLUSIONES}

Las muestras analizadas en este estudio no sobrepasaron los límites máximos de residuos permitidos establecidos en el Codex alimentarius para hortalizas, por lo que no representan algún riesgo para la salud del consumidor, ni para su comercialización.

Se sugiere que se realice un monitoreo continuo de plaguicidas en las hortalizas analizadas para asegurar su inocuidad alimentaria en relación a estos contaminantes que permita establecer políticas de comercialización, manejo de plaguicidas, así como una disminución de riesgos tanto para el consumidor local, regional e internacional; así como incluir otros grupos de plaguicidas.

\section{AGRADECIMIENTOS}

Al Fondo Sectorial-CONACYT: Convocatoria SEMARNAT- S0010-2008-1 por el financiamiento del estudio.

\section{REFERENCIAS}

Atreya, N. 2006. Chemophobia-Pesticide residues in food. Outlooks on Pest Management. 17- 24.

Acuña, G. 2008. Determinación de plaguicidas organoclorados en suelos de comunidades urbanas en el sur de sonora. Tesis de Licenciatura. Instituto Tecnológico de Sonora, Ciudad Obregón, Sonora, México. 68pp.

Agencia para Sustancias Tóxicas y el Registro de Enfermedades. (ATSDR). 2002. Reseña Toxicológica del Metoxicloro (edición actualizada) (en inglés). Atlanta, GA: Departamento de Salud y Servicios Humanos de EE.UU. Servicio de Salud Pública.

Aldana-Madrid, M.L., Valdez-Hurtado, S., Vargas-Valdez, N.D., Salazar-López, N.J., Silveira-Gramont, M.I., Loarca-Piña, F.G., Rodríguez-Olibarria, G., Wong- Corral, F.J., Borboa-Flores, E. y Burgos-Hernández, A. 2008. Insecticide residues in stored grains in Sonora, Mexico: quantification and toxicity testing. Bulletin Of Environmental-Contamination and Toxicology. 80 (2): 93-96. 
Bejarano-González, F. 2017. Los plaguicidas altamente peligrosos nuevo tema normativo internacional y su perfil nacional en México. En: Los plaguicidas altamente peligrosos en México. (Bejarano-González F. Ed.). pp 10-96. RAPAM, CIAD, UAEMEX, INIFAP, UCCS, IPEN, PNUD. México.

Benítez-Trinidad, A. B., Herrera-Moreno, J. F., XotlanihuaGervacio, M. C., Bernal-Hernández, Y. Y., Medina-Díaz, I. M., Barrón-Vivanco, B. S., González-Arias, C. A., Rojas-García, A. E. 2018. Patrón de uso de plaguicidas y biomarcadores bioquímicos en una población de fumigadores urbanos. Revista Internacional de Contaminación Ambiental. 34 (Especial sobre Contaminación y Toxicología por Plaguicidas): 61-71.

Borchers, A., Teuber, S., Keen, C. L. y Gershwin, M. E. 2010. Food Safety. Clinical Reviews in Allergy and Immunology. 39: 95141.

Cantú-Soto, E.U., Meza-Montenegro, M.M., Valenzuela-Quintanar, A.I., Félix-Fuentes, A., Grajeda-Cota, P., Balderas-Cortes, J.J., Osorio-Rosas, C.L., Acuña-García, G., y Aguilar-Apodaca, M.G. 2011. Residues of Organochlorine Pesticides in Soils from the Southern Sonora, Mexico. Bulletin of Environmental Contamination and Toxicology. 87 (5): 556-560.

CESCCO-IHISE. 1997. Residuos de plaguicidas en hortalizas de mercados y supermercados de Tegucigalpa. Tegucigalpa, CESCCO-IHISE. 106 pp.

Codex alimentarius-FAO-OMS. [Consultado 2 julio 2018]. Disponible en: http://www.fao.org/fao-whocodexalimentarius/about-codex/es/\#c453328

De Boer, J. y Wester, P. 1991. Chlorobiphenyls and organochlorine pesticides in various sub-Antartic Organisms. Marine Pollution Bulletin. 22: 441.

Fenik, J., Tankiewicz, M. y Biziuk, M. 2011. Properties and determination of pesticides in fruits and vegetables. Trends in Analytical Chemistry. 30: 814-826.

Galt, E. R. 2009. Overlap of US FDA residue tests and pesticides used on imported vegetables: Empirical fndings and policy recommendations. Food Policy. 34: 468-476.

García-Hernández, J. 2018. Estado actual de la investigación sobre plaguicidas en México. Revista Internacional de Contaminación Ambiental. 34 (Especial sobre Contaminación y Toxicología por Plaguicidas): 29-60.

García-Hernández, J., Leyva-García, G. y Aguilera-Márquez, D. 2017. Los plaguicidas altamente peligrosos en el Valle del Yaqui, Sonora. En: Los plaguicidas altamente peligrosos en México. (Bejarano-González F. Ed.). pp: 173-183. RAPAM, CIAD, Red Temática de Toxicología, UAEMEX, INIFAP, UCCS, IPEN, PNUD. México.

Giannuzzi, L. 1994. Residuos de plaguicidas organoclorados en papas que se comercializan en la Ciudad de La Plata y Gran La Plata (Argentina). 103-112.

González-Arias, C.A., Robledo-Marenco, M.L., Medina-Díaz, I.M., Velázquez-Fernández, J.B., Girón-Pérez, M.I., QuintanillaVega, B., Ostrosky-Wegman, P., Pérez-Herrera, N.E. y RojasGarcía, A.E. 2010. Patrón de uso y venta de plaguicidas en Nayarit, México. Revista Internacional de Contaminación Ambiental. 26 (3): 221-228.

Koepke, R., Warne,-M., Petreas, M., Cabria, A., Danis, R., Hernández, M. y Eskenasy, B. 2004. Serum DDT and DDE levels in pregnant women of Chiapas, Mexico. Archives of Environmental Health. 59: 559-565.
Leal-Soto, S. D., Valenzuela-Quintanar, A. I., Gutiérrez- Coronado, M. L., Bermúdez-Almada, M.C., García- Hernández, J., Aldana Madrid, M.L., Grajeda-Cota, P., Silveira-Gramont, M.I., MezaMontenegro, M.M., Palma-Durán, S.A., Leyva-García, G.N., Camarena-Gómez, B.O. y Valenzuela-Navarro C.P. 2014. Residuos de plaguicidas organoclorados en suelos agrícolas. Terra Latinoamericana. 32:1-11.

Limón-Miro, A. T., Aldana-Madrid, M. L., Alvarez-Hernandez, G., Antunez-Roman, L. E., Rodriguez-Olibarria, G., Valencia Juillerat, M.E. 2017. Breast milk intake and mother to infant pesticide transfer measured by deuterium oxide dilution in agricultural and urban areas of Mexico. Chemosphere. (181): 682-689.

Leyva-Morales, J.B., García-de la Parra, L.M., Bastidas-Bastidas, P.J., Astorga-Rodríguez, J.E., Bejarano-Trujillo, J., CruzHernández, A., Martínez-Rodríguez, I.E. y BetancourtLozano, M. 2014. Uso de plaguicidas en un valle agrícola tecnificado en el noroeste de México. Revista Internacional de Contaminación Ambiental. 30 (3): 247-261.

Miglioranza, K. S., González Sagrario, M.A., Aizpún de Moreno, J.E., Moreno, V.J., Escalante, A.H. y Osterrieth, M.L. 2002. Agricultural soil as a potential source of input of organochlorine pesticides into nearby pond. Environmental Science and Pollution Research. 9 (4): 250-256.

Meza, M.M., Valenzuela, A. I., Balderas, J. J., Yañez, L., Gutiérrez, M. L., Cuevas A., Gandolfi, A.J. 2013. Exposure assessment of organochlorine pesticides, arsenic, and lead in children from the major agricultural areas in Sonora, Mexico. Archives of Environmental Contamination and Toxicology. 64: 519-527.

Moreno, E. 2008. Determinación cuantitativa de piretroides en suelo y agua de las zonas agrícolas y urbanas de los Valles del Yaqui y Mayo. Tesis de Maestría. Universidad de Sonora, Hermosillo, Sonora, México.

Moreno, M. J. A. y López, L. M. G. 2005. Desarrollo agrícola y uso de agroquímicos en el Valle de Mexicali. Estudios Fronterizos. 6: 119-153.

Navarro, M. J. 2012. Plaguicidas organoclorados residuales en suelos agrícolas de los valles del Yaqui y Mayo, Sonora. Tesis de licenciatura. Instituto Tecnológico de Sonora. Ciudad Obregón, Sonora, México.

Nolasco, S. C. y Zúniga, N. 1992. Uso y Manejo de Plaguicidas en una Zona Agrícola. Lepaterique. Estudio de Campo y Verificación Analítica. Facultad de Química y Farmacia. Tegucigalpa, UNAH: 154.

Ortiz, M., Yáñez, L. y Díaz-Barriga, F. 2001. Comportamiento ambiental del DDT. Laboratorio de Toxicología Ambiental. Facultad de Medicina Universidad Autónoma de San Luis Potosí.

Osorio, C. 2008. Determinación de plaguicidas organoclorados en suelos de comunidades rurales en el valle del Yaqui y Mayo, Sonora, México. Tesis de licenciatura. Instituto Tecnológico de Sonora. Ciudad Obregón, Sonora, México.

Pérez, M. A., Navarro, H. y Miranda, E. 2013. Residuos de plaguicidas en hortalizas: problemática y riesgo en México. Revista Internacional de Contaminación Ambiental. 29 (Número especial sobre plaguicidas): 45-64.

Pérez, M. A., Segura, A., García, R., Colinas, T., Pérez, M., Vázquez, A. y Navarro, H. 2009. Residuos de plaguicidas organofosforados en cabezuela de brócoli (Brassica oleracea) determinados por cromatografía de gases. Revista Internacional de Contaminación Ambiental. 25: 103-110. 
Pirsaheb., M., Limoee, M., Namdari, F. y Khamutian, R. 2015. Organochlorine pesticides residue in breast milk: a systematic review. Medical Journal of The Islamic Republic of Iran. 29: 228.

Ramírez-Milla,L.G. 2009. Determinación de pesticidas en vegetales mediante cromatografía de gases-espectrometría de masa/masa (GC-MS/MS). Tesis de licenciatura. Universidad Tecnológica de la Mixteca. Huajuapan de León, Oaxaca.

Rascón, A. 2011. Determinación de los niveles de plaguicidas organoclorados en suelo residencial y agrícola de la comunidad Yaqui Tetabiate, Sonora. Tesis de Licenciatura. Instituto Tecnológico de Sonora, Ciudad Obregón, Sonora, México.

Romero Hernández, J.A., Amaya Chávez, A., Miranda Rivera, M.G. y García Fabila, M.M. 2018. Métodos cromatográficos para la determinación de endosulfán en alimentos. Revista Internacional de Contaminación Ambiental. 34 (Especial sobre Contaminación y Toxicología por Plaguicidas): 81-94.

USEPA. 1997. Annual spray drift review. Office of Pesticide Programs, Environmental Fate and Effects Division, United States Environmental Protection Agency. [Consultado 28 junio 2018]. http://www.epa.gov/scipoly/sap/ meetings/1997/december/spraydrift.htm 17/04/2017

Valenzuela, A.I., Camarena, B.O., Gutiérrez, M.L., Grajeda, P., Silveira, M.I., Aldana, M.L., Meza, M.M. y García, J. 2015. Desarrollo de una metodología que permita llevar a cabo el inventario, caracterización y verificación de sitios potencialmente contaminados con compuestos orgánicos persistentes en las principales zonas agrícolas del estado de Sonora. Informe Técnico Final-CONACYT.

Valenzuela, A.I., Ortega, M.I., Ballesteros, M.N., Gutiérrez, M.L., Grajeda P., Cabrera, R. M., Saucedo; S., Contreras, A. 2008. Evaluación directa a plaguicidas de jornaleros agrícolas e indirecta por consumo de agua y alimentos y su impacto en la expresión del síndrome metabólico. Informe Técnico Final- CONACYT.

Valenzuela-Quintanar, A. I., Armenta-Corral, R., Moreno- Villa, E., Gutiérrez-Coronado, L., Grajeda-Cota, P. y Orantes-Arenas, C. 2006. Optimization and validation of a method based on matrix solid-phase dispersion for organophosphorus pesticides in vegetables. Revista de la Facultad de Agronomía (LUZ). 23 (4): 460-470.

Van derHoff,G.R.y vanZoonen,P.1999.Traceanalysis of pesticides by gas chromatography. Journal of Chromatography A. 843: 301-332.

Verdugo, A. A. 2012. Cuantificación de los niveles de plaguicidas organoclorados en agua superficial y subterránea de áreas agrícolas del sur de Sonora. Tesis de Licenciatura. Instituto Tecnológico de Sonora, Ciudad Obregón, Sonora México.

Waliszewski, S. M., Carvajal, O., Gómez-Arroyo, S., AmadorMuñuz, O., Villalobos-Pietrini, R., Hayward-Jones, P.M. y Valencia-Quintana, R. 2008. DDT and $\mathrm{HCH}$ isomer levels in soils, carrot root and carrot leaf samples. Bulletin of Environmental Contamination and Toxicology. 81: 343-347. 